\title{
Usefulness of Upstroke Time per Cardiac Cycle for Cardiovascular and All-Cause Mortality Prediction in Patients with Normal Ankle- Brachial Index
}

\author{
Ho-Ming Su ${ }^{1,2,3}$, Wen-Hsien Lee ${ }^{1,2,3}$, Wei-Chung Tsai ${ }^{1,3}$, Tzu-Chieh Lin ${ }^{1}$, Ye-Hsu Lu ${ }^{1}$, Chee-Siong Lee ${ }^{1,3}$, \\ Tsung-Hsien Lin ${ }^{1,3}$, Wen-Chol Voon ${ }^{1,3}$, Wen-Ter Lai ${ }^{1,3}$, Sheng-Hsiung Sheu ${ }^{1,3}$ and Po-Chao Hsu ${ }^{1,3}$ \\ ${ }^{1}$ Division of Cardiology, Department of Internal Medicine, Kaohsiung Medical University Hospital, Kaohsiung, Taiwan \\ ${ }^{2}$ Department of Internal Medicine, Kaohsiung Municipal Siaogang Hospital, Kaohsiung, Taiwan \\ ${ }^{3}$ Faculty of Medicine, College of Medicine, Kaohsiung Medical University, Kaohsiung, Taiwan
}

Aim: Abnormal ankle-brachial index (ABI) is regarded as peripheral artery disease and can be used to predict cardiovascular $(\mathrm{CV})$ outcomes. However, the usefulness of $\mathrm{ABI}$ for the prediction of $\mathrm{CV}$ outcome in patients with normal ABI is limited. Upstroke time per cardiac cycle (UTCC) is recently reported to be associated with mortality in patients with acute myocardial infarction and the elderly. Therefore, we aimed to evaluate UTCC, left ventricular ejection fraction (LVEF), brachial-ankle pulse wave velocity (baPWV), and ABI for the prediction of mortality in patients with normal ABI.

Methods: Patients arranged for echocardiographic examinations were enrolled, and 1076 patients with normal ABI were included. ABI, baPWV, and UTCC were measured by an ABI-form device.

Results: The median follow-up to mortality was 95 months. There were $88 \mathrm{CV}$ and 244 all-cause deaths. After multivariate analysis, UTCC was associated with increased CV and all-cause mortality $(P \leq 0.004)$. Age, diabetes, heart failure, left ventricular hypertrophy, baPWV, and LVEF were also independent predictors of CV and allcause mortality, but ABI was not. Furthermore, UTCC had a better additive predictive value than ABI, baPWV, and LVEF for CV mortality $(P \leq 0.012)$. It also had a better additive predictive value than ABI and LVEF for allcause mortality $(P \leq 0.013)$.

Conclusions: UTCC is an independent predictor for CV and all-cause mortality in patients with normal ABI. It also has a better additive predictive value of $\mathrm{CV}$ and all-cause mortality than $\mathrm{ABI}$ and LVEF. Therefore, UTCC is a simple, novel, and useful parameter for identifying high-risk patients with normal ABI.

Key words: Ankle-brachial index, All-cause mortality, Cardiovascular, Mortality, Left ventricular ejection fraction, Upstroke time per cardiac cycle

\section{Introduction}

Ankle-brachial index (ABI) is a useful diagnostic tool for peripheral arterial disease $(\mathrm{PAD})^{1,2)}$. ABI of both $<0.9$ and $>1.4$ is considered abnormal ABI and was reported to be associated with poor cardiovascular $(\mathrm{CV})$ outcome ${ }^{3-10)}$. ABI within 0.9 and 1 is regarded as borderline $\mathrm{ABI}^{3)}$. Conversely, $\mathrm{ABI}$ within 1 and 1.4 is regarded as normal $\mathrm{ABI}$ and has better long-term
$\mathrm{CV}$ outcome than abnormal $\mathrm{ABI}^{3,11-13)}$. However, there are only a few studies about the prediction of long-term $\mathrm{CV}$ and all-cause mortality in patients with normal ABI, and the usefulness of ABI for the prediction of mortality in this patient group is limited.

Upstroke time (UT) calculated from the initial of the systolic rise to the peak of the pulse wave in the lower limb was shown to be an excellent indicator of peripheral artery disease (PAD) ${ }^{14-16)}$. Recently, UT per

Address for correspondence: Po-Chao Hsu, Division of Cardiology, Department of Internal Medicine; Kaohsiung Medical University Hospital, 100 Tzyou 1st Road, Kaohsiung. 80708, Taiwan, ROC E-mail: pochao.hsu@gmail.com

Received: September 28, 2020 Accepted for publication: December 23, 2020

Copyright@2021 Japan Atherosclerosis Society

This article is distributed under the terms of the latest version of CC BY-NC-SA defined by the Creative Commons Attribution License. 
cardiac cycle (UTCC) was reported to be a diagnostic tool of PAD and a predictor of mortality in patients with acute myocardial infarction and older population ${ }^{17,18)}$. Therefore, our study aimed to evaluate the usefulness of UTCC for the prediction of CV and all-cause mortality in patients with normal ABI. Additionally, we compared UTCC with other important parameters such as left ventricular ejection fraction (LVEF), brachial-ankle pulse wave velocity (baPWV), and ABI to see whether UTCC had a better predictive value of $\mathrm{CV}$ and all-cause mortality.

\section{Methods}

\section{Study Population and Design}

Study population was enrolled from the patients arranged for echocardiographic examinations at Kaohsiung Municipal Siaogang Hospital from 2010 to 2012. Our inclusion criterion was patient with normal $A B I(1<A B I<1.4)$. Patients with significant atrial fibrillation and diseases of mitral and aortic valves were excluded. Finally, 1076 patients were included in our study. This study was approved by the institutional review board committee of the Kaohsiung Medical University Hospital (KMUHIRB). We acquired informed consent from the patients and conducted our study according to the declaration of Helsinki.

Assessment of UTCC, baPWV, and ABI by ABIform Device

We measured UTCC and ABI by ABI-form device (VP1000, Japan), which measures blood pressures of four limbs via oscillometric method ${ }^{19,20)}$, and it can also obtain the data of ABI, baPWV, and UT at the same time. UT was shown by the device as the pulse foot-to-peak transit time. UTCC was calculated as the UT divided by cardiac cycle* $100 \%{ }^{17,21)}$. After we acquired the UTCC of the bilateral foot, the higher one was selected as our data. For measuring baPWV, pulse waves that were acquired from the brachial and tibial arteries were recorded simultaneously, and the transmission time, which was defined as the time interval between the initial increase in brachial and tibial waveforms, was determined. The transmission distance from the arm to each ankle was calculated according to body height. The value of baPWV was automatically calculated as the transmission distance divided by the transmission time. ABI was calculated by the ratio of the lower ankle over the higher brachial systolic blood pressure. We performed measurement of the ABI-form device in each subject within the same day of echocardiographic examination.

\section{Echocardiographic Examination}

All echocardiographic examinations were conducted by a single experienced cardiologist. The cardiologist was blinded to the clinical data. The LVEF was measured by the Simpson method.

\section{Definition of CV and All-Cause Mortality}

We followed our patients till December 2018 and acquired survival information and causes of death from the official death certificate and final confirmation by the Ministry of Health and Welfare. The causes of death were classified by the International Classification of Diseases 10th Revision. Causes of CV mortality were defined as deaths due to cerebral vascular disease, ischemic heart disease, myocardial infarction, heart failure, valvular heart disease, and atherosclerotic vascular disease.

\section{Collection of Demographic and Medical data}

Demographic and medical data including age, gender, smoking history, and comorbid conditions were obtained from interviews or medical records of patients. The body mass index was calculated as the ratio of weight in kilograms divided by the square of height in meters. Heart failure was defined as left ventricular systolic dysfunction with a left ventricular ejection fraction of $\leqq 40 \%$ or having a known history of heart failure. Hypertension was defined as systolic blood pressure of $\geqq 140 \mathrm{mmHg}$ or diastolic blood pressure of $\geqq 90 \mathrm{mmHg}$, or anti-hypertensive drugs were prescribed. Diabetes was defined as a fasting blood glucose level of $>126 \mathrm{mg} / \mathrm{dL}$, or hypoglycemic agents were used to controlling blood glucose levels. Dyslipidemia was defined as total cholesterol of $\geqq 200$ $\mathrm{mg} / \mathrm{dL}$ or low-density lipoprotein of $\geqq 130 \mathrm{mg} / \mathrm{dL}$. Additionally, medications of patients including aspirin, beta blockers, calcium channel blockers, angiotensin converting enzyme inhibitors, angiotensin II receptor blockers, and diuretics during the study period were obtained from medical records.

\section{Statistical Analysis}

SPSS 22.0 was used to conduct statistical analyses. Our data were shown as a percentage or mean \pm standard deviation. Categorical variables were compared using the chi-square test. Continuous variables were compared using an independent sample $t$-test. We selected significant variables in our univariate analysis into multivariate analysis. We adjusted significant variables and time to mortality using Cox regression analysis. We also calculated the improvement of global chi-square to evaluate the additive value of ABI, baPWV, LVEF, and UTCC over a basic model for long-term $\mathrm{CV}$ and all-cause 
Table 1. Comparison of clinical characteristics between patients with and without mortality

\begin{tabular}{lccr}
\hline Baseline Characteristics & Mortality (-) & Mortality (+) & $P$ value \\
\hline Number & 832 & 244 & \\
Age (yr) & $58 \pm 12$ & $68 \pm 13$ & $<0.001$ \\
Male gender (\%) & $54.0 \%$ & $63.0 \%$ & 0.015 \\
Smoking (\%) & $15.7 \%$ & $11.8 \%$ & 0.230 \\
Diabetes (\%) & $21.4 \%$ & $42.2 \%$ & $<0.001$ \\
Hypertension (\%) & $68.1 \%$ & $70.1 \%$ & 0.584 \\
Dyslipidemia (\%) & $60.1 \%$ & $54.0 \%$ & 0.141 \\
Coronary artery disease (\%) & $16.2 \%$ & $20.2 \%$ & 0.175 \\
Heart failure (\%) & $3.1 \%$ & $19.4 \%$ & $<0.001$ \\
LVH (\%) & $53.0 \%$ & $74.8 \%$ & $<0.001$ \\
Body mass index (kg/m $\left.{ }^{2}\right)$ & $26.3 \pm 3.7$ & $25.8 \pm 4.3$ & 0.076 \\
ABI & $1.14 \pm 0.06$ & $1.14 \pm 0.07$ & 0.481 \\
baPWV (cm/s) & $1666 \pm 345$ & $1995 \pm 517$ & $<0.001$ \\
LVEF (\%) & $65.6 \pm 10.9$ & $57.6 \pm 16.2$ & $<0.001$ \\
UTCC (\%) & $16.1 \pm 2.8$ & $17.9 \pm 3.6$ & $<0.001$ \\
Medication & & & \\
$\quad$ Aspirin (\%) & $31.8 \%$ & $30.8 \%$ & 0.853 \\
$\beta$-blockers (\%) & $41.0 \%$ & $44.9 \%$ & 0.301 \\
CCBs (\%) & $35.5 \%$ & $35.0 \%$ & 0.939 \\
ACEIs (\%) & $9.0 \%$ & $11.9 \%$ & 0.177 \\
ARBs (\%) & $43.9 \%$ & $43.6 \%$ & 0.942 \\
Diuretics (\%) & $23.6 \%$ & $33.1 \%$ & 0.004 \\
\hline Abbrevions: ABI $\%$ & $c 01 \%$ & \\
\hline
\end{tabular}

Abbreviations: ABI, ankle-brachial index; ACEI, angiotensin converting enzyme inhibitor; ARB, angiotensin II receptor blocker; baPWV, brachial-ankle pulse wave velocity; CCB, calcium channel blocker; LVEF, left ventricular ejection fraction; UTCC, upstroke time per cardiac cycle

mortality prediction. Kaplan-Meier survival curve was calculated from baseline to time of mortality events. A $P$ value of $<0.05$ was considered statistically significant.

\section{Results}

Among the 1076 subjects, the median follow-up to mortality was 95 months $(25$ th -75 th percentile: $87-101$ months). The mean age in our study was $60.4 \pm 13.2$ years. There were $88 \mathrm{CV}$ and 244 all-cause deaths.

Table 1 shows the clinical characteristics of patients with and without mortality. Patients with mortality had older age, male gender, higher percentage of diabetes, heart failure, left ventricular hypertrophy, higher baPWV $(1995 \pm 517$ versus $1666 \pm 345, P<0.001)$, lower LVEF $(57.6 \pm 16.2$ versus $65.6 \pm 10.9, P<0.001)$, higher UTCC (17.9 \pm 3.6 versus $16.1 \pm 2.8, P<0.001)$, and higher percentage of diuretic use compared with patients without mortality. There was no significant difference between the percentage of smoking, hypertension, dyslipidemia, body mass index, and medication use (aspirin/beta blocker/calcium channel blocker use/ angiotensin converting enzyme inhibitor/angiotensin II receptor blocker).

Table 2 displays the predictors of $\mathrm{CV}$ mortality using Cox regression analysis. Age, diabetes, dyslipidemia, coronary artery disease, heart failure, left ventricular hypertrophy, baPWV, LVEF, and UTCC are significant predictors of $\mathrm{CV}$ mortality in the univariate analysis. After multivariate analysis, age, diabetes, heart failure, left ventricular hypertrophy, baPWV, LVEF (hazard ratio $[\mathrm{HR}]=0.950 ; 95 \%$ confidence interval $[\mathrm{CI}]: 0.926-0.976 ; P<0.001$ ), and UTCC $(\mathrm{HR}=1.134 ; 95 \% \mathrm{CI}: 1.041-1.236$; $P=0.004)$ were significantly associated with $\mathrm{CV}$ mortality.

Because chronic kidney disease is also strongly associated with PAD, we further added the variable of estimated Glomerular filtration rate into multivariate analysis for CV mortality. Results showed that age, heart failure, left ventricular hypertrophy, LVEF $(\mathrm{HR}=0.953$; 95\% CI: 0.929-0.977; $P<0.001)$, and UTCC $(\mathrm{HR}=1.139 ; 95 \% \mathrm{CI}: 1.039-1.248 ; P=0.005)$ were significantly associated with $\mathrm{CV}$ mortality, but estimated Glomerular filtration was not $(P=0.482)$.

Table 3 displays the predictors of all-cause mortality using Cox regression analysis. Age, diabetes, 
Su et al.

Table 2. Predictors of cardiovascular mortality using Cox regression analysis

\begin{tabular}{|c|c|c|c|c|}
\hline Parameter & \multicolumn{2}{|c|}{ Univariate (CV mortality) } & \multicolumn{2}{|c|}{ Multivariate (CV mortality) } \\
\hline Age (yr) & $1.078(1.052-1.104)$ & $<0.001$ & $1.063(1.031-1.095)$ & $<0.001$ \\
\hline Male gender & $1.153(0.683-1.944)$ & 0.594 & & \\
\hline Hypertension (Yes or No) & $1.020(0.580-1.792)$ & 0.946 & & \\
\hline Diabetes (Yes or No) & $2.823(1.691-4.711)$ & $<0.001$ & $1.880(1.023-3.457)$ & 0.042 \\
\hline Coronary artery disease (Yes or No) & $2.140(1.218-3.761)$ & 0.008 & - & 0.265 \\
\hline Heart failure (Yes or No) & $7.760(4.308-13.978)$ & $<0.001$ & $2.495(1.066-5.836)$ & 0.035 \\
\hline LVH (Yes or No) & $4.159(2.043-8.467)$ & $<0.001$ & $2.346(1.084-5.077)$ & 0.030 \\
\hline Smoking (ever vs no) & $0.645(0.277-1.501)$ & 0.309 & & \\
\hline Body mass index (per kg/m²) & $0.955(0.890-1.025)$ & 0.202 & & \\
\hline $\mathrm{ABI}$ (per 1SD) & $0.033(0.001-1.661)$ & 0.088 & & \\
\hline \multicolumn{5}{|l|}{ Medications } \\
\hline Aspirin use & $1.246(0.731-2.124)$ & 0.419 & & \\
\hline Beta blocker use & $1.177(0.704-1.968)$ & 0.535 & & \\
\hline Calcium channel blocker use & $1.092(0.650-1.836)$ & 0.739 & & \\
\hline ACEI use & $1.242(0.534-2.891)$ & 0.614 & & \\
\hline ARB use & $1.520(0.907-2.548)$ & 0.112 & & \\
\hline Diuretic use & $1.219(0.700-2.122)$ & 0.484 & & \\
\hline
\end{tabular}

CI: confidence interval; CV: cardiovascular; HR: hazard ratio; SD: standard deviation; other abbreviations as in Table 1.

Table 3. Predictors of all-cause mortality using Cox regression analysis

\begin{tabular}{|c|c|c|c|c|}
\hline Parameter & \multicolumn{2}{|c|}{ Univariate (all-cause mortality) } & \multicolumn{2}{|c|}{ Multivariate (all-cause mortality) } \\
\hline Male gender & $1.279(0.932-1.754)$ & 0.127 & & \\
\hline Hypertension (Yes or No) & $1.046(0.743-1.471)$ & 0.797 & & \\
\hline Diabetes (Yes or No) & $2.331(1.709-3.181)$ & $<0.001$ & $1.840(1.277-2.650)$ & 0.001 \\
\hline Coronary artery disease (Yes or No) & $1.288(0.875-1.896)$ & 0.200 & & \\
\hline Heart failure (Yes or No) & $4.639(3.084-6.978)$ & $<0.001$ & $1.820(1.006-3.293)$ & 0.048 \\
\hline LVH (Yes or No) & $2.119(1.489-3.015)$ & $<0.001$ & $1.498(1.001-2.243)$ & 0.049 \\
\hline Smoking (ever vs no) & $0.798(0.500-1.275)$ & 0.346 & & \\
\hline Body mass index $\left(\right.$ per $\mathrm{kg} / \mathrm{m}^{2}$ ) & $0.951(0.911-0.993)$ & 0.022 & - & 0.972 \\
\hline \multicolumn{5}{|l|}{ Medications } \\
\hline Aspirin use & $0.970(0.694-1.357)$ & 0.860 & & \\
\hline Beta blocker use & $1.107(0.811-1.512)$ & 0.521 & & \\
\hline Calcium channel blocker use & $0.935(0.680-1.287)$ & 0.681 & & \\
\hline ACEI use & $0.883(0.490-1.590)$ & 0.678 & & \\
\hline $\mathrm{ARB}$ use & $0.937(0.688-1.277)$ & 0.680 & & \\
\hline Diuretic use & $1.380(0.996-1.914)$ & 0.053 & & \\
\hline
\end{tabular}

CI: confidence interval; HR: hazard ratio; SD: standard deviation; other abbreviations as in Table 1.

Advance Publication Journal of Atherosclerosis and Thrombosis 
(A)

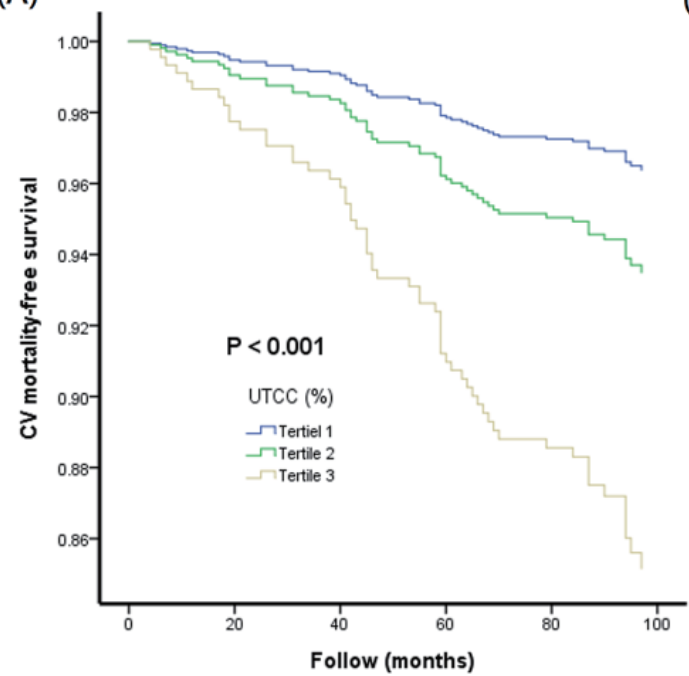

(B)

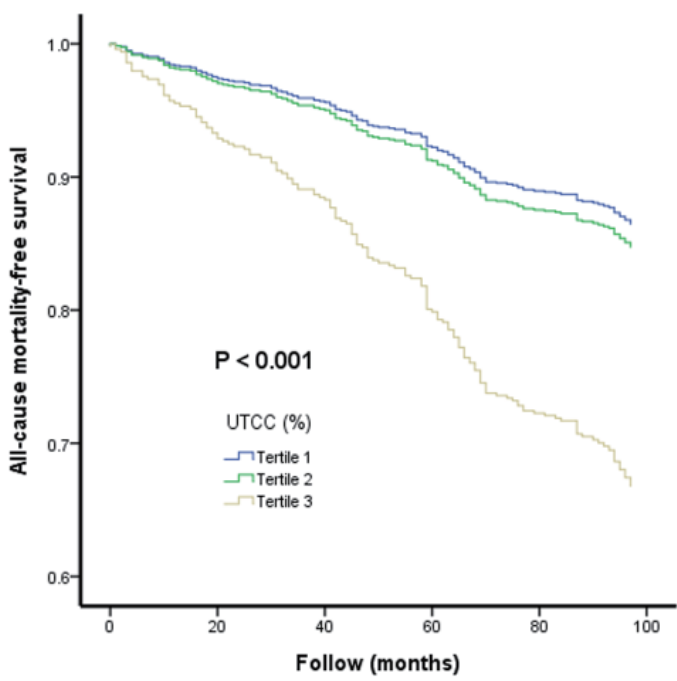

Fig. 1. Kaplan-Meier curves of UTCC divided into tertile for CV (Fig. 1A) and all-cause mortality-free survival (Fig. 1B)

Abbreviation: CV, cardiovascular; UTCC, upstroke time per cardiac cycle

dyslipidemia, heart failure, left ventricular hypertrophy, body mass index, baPWV, LVEF, and UTCC are significant predictors of all-cause mortality in the univariate analysis. After multivariate analysis, age, diabetes, heart failure, left ventricular hypertrophy, baPWV, LVEF $(\mathrm{HR}=0.965 ; 95 \% \mathrm{CI}$ : $0.950-0.981 ; P<0.001)$, and UTCC $(\mathrm{HR}=1.084$; 95\% CI: $1.028-1.143 ; P=0.003)$ were significantly associated with all-cause mortality.

Fig. 1 shows the Kaplan-Meier curves of UTCC divided into tertile for $\mathrm{CV}$ (Fig. 1A) and all-cause mortality-free survival (Fig. 1B) (both $P<0.001$ ). Tertile 1 included UTCC of $<15 \%$. Tertile 2 included UTCC within $15 \%$ and $17.5 \%$. Tertile 3 included UTCC of $>17.5 \%$.

Fig. 2 illustrates the Nested Cox model for CV (Fig. 2A) and all-cause mortality (Fig. 2B) prediction. The basic model in Fig. 2A included age, diabetes, dyslipidemia, coronary artery disease, heart failure, and left ventricular hypertrophy. After adding ABI, baPWV, LVEF, and UTCC into the basic model respectively, we found that basic model+baPWV, basic model + UTCC, and basic model + LVEF had better predictive values for $\mathrm{CV}$ mortality than the basic model alone $(P \leq 0.010)$. Basic model + UTCC also had better a predictive value than basic model + ABI $(P<0.001)$, basic model + baPWV $(P=0.005)$, and basic model $+\operatorname{LVEF}(P=0.012)$. Additionally, basic model + LVEF + UTCC had better predictive values for $\mathrm{CV}$ mortality than basic model + LVEF $(P$ $<0.001)$. Basic model + LVEF + UTCC also had a better predictive value than basic model $+\mathrm{LVEF}+\mathrm{ABI}$ for $\mathrm{CV}$ mortality $(P=0.002)$. The basic model in
Fig. 2B included age, diabetes, dyslipidemia, heart failure, left ventricular hypertrophy, and body mass index. After adding ABI, baPWV, LVEF, and UTCC into the basic model respectively, we found that basic model + baPWV, basic model + UTCC, and basic model + LVEF had better predictive values for allcause mortality than the basic model alone $(P<$ 0.001). Basic model + UTCC also had a better predictive value than basic model $+\operatorname{LVEF}(P=0.013)$. Furthermore, basic model + LVEF + UTCC had a better predictive value for all-cause mortality than basic model + LVEF $(P<0.001)$. Basic model + LVEF + UTCC also had a better predictive value than basic model + LVEF + ABI for all-cause mortality $(P<$ 0.001).

\section{Discussion}

Our study aimed to evaluate the usefulness of UTCC for the prediction of CV and all-cause mortality in patients with normal ABI, and we found several major findings in the present study. First, higher UTCC was significantly associated with increased CV and overall mortality. Second, age, diabetes, heart failure, left ventricular hypertrophy, baPWV, and LVEF were also significant predictors of $\mathrm{CV}$ and all-cause mortality in this patient group. Third, UTCC had a better additive predictive value than $\mathrm{ABI}$, baPWV, and LVEF for CV mortality. It also had better additive predictive than ABI and LVEF for all-cause mortality.

Abnormal ABI is not only regarded as a diagnostic indicator of PAD but also associated with 

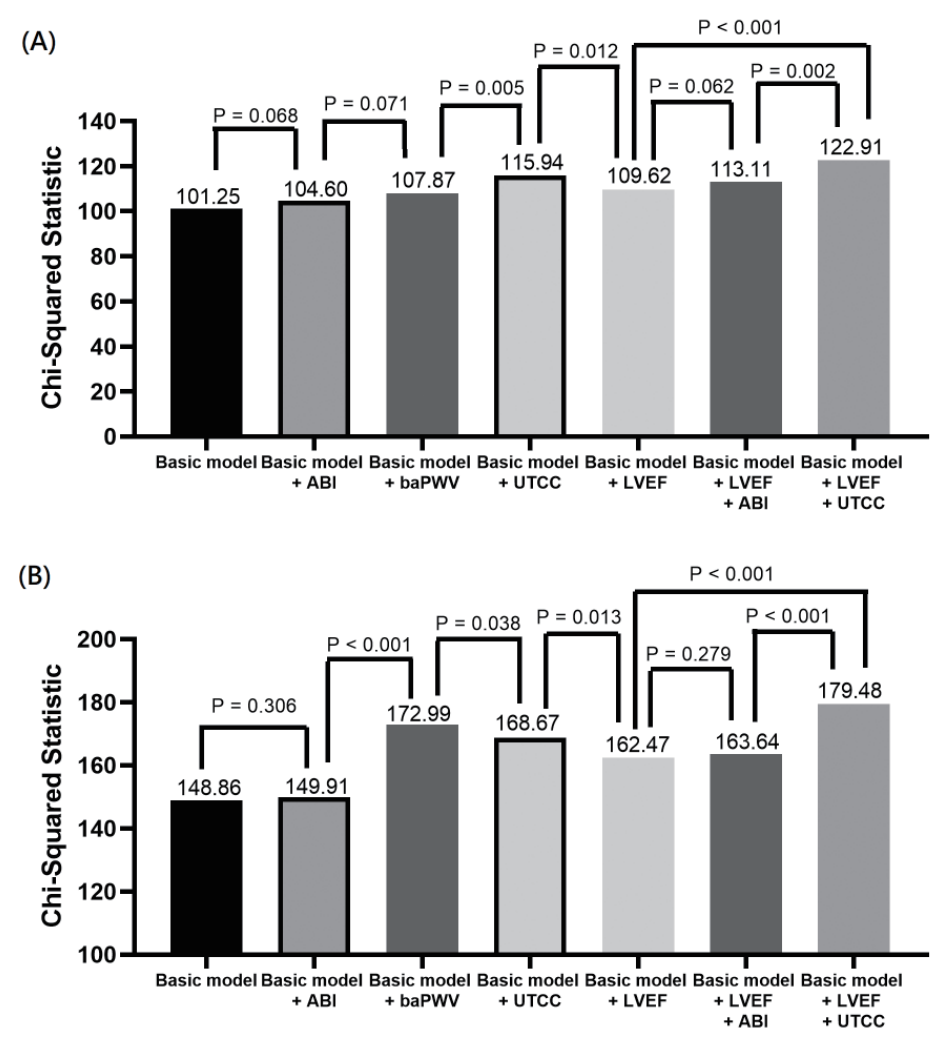

Fig. 2. The nested Cox model for CV (Fig. 2A) and all-cause mortality (Fig. 2B) prediction by calculating the improvement in global chi-square value

The basic model in Fig. 2A included age, diabetes, dyslipidemia, coronary artery disease, heart failure, and left ventricular hypertrophy. The basic model in Fig. 2B included age, diabetes, dyslipidemia, heart failure, left ventricular hypertrophy, and body mass index.

Abbreviation: ABI, ankle-brachial index; baPWV, brachial-ankle pulse wave velocity; LVEF, left ventricular ejection fraction; UTCC, upstroke time per cardiac cycle

higher $\mathrm{CV}$ and all-cause mortality ${ }^{3-10)}$. Compared with patients with abnormal ABI, those with normal $\mathrm{ABI}$ had better CV outcomes and lower morbidity and mortality ${ }^{11-13)}$. Although ABI is a useful predictor of future CV outcome in several populations such as the general population, patients with diabetes, hypertension, chronic kidney disease, dialysis, stroke, and so on ${ }^{6-13)}$, the usefulness of ABI for the prediction of long-term $\mathrm{CV}$ and all-cause mortality in patients with normal ABI is limited.

An ABI-form device (VP 1000) could automatically measure blood pressures of four limbs via oscillometric $\operatorname{method}^{19,20)}$. It could also acquire the values of $\mathrm{ABI}$ and UT at the same time. According to the literature, UT plays an important role in the diagnosis of PAD, and it is also significantly correlated with $A B I^{17,21)}$. In recent years, $\mathrm{Yu} \mathrm{S}$ et al. reported that UTCC was significantly correlated with renal and vascular damage in the old Chinese population ${ }^{21)}$. Sheng CS et al. showed that UTCC was equivalent to ABI for the diagnosis of PAD and can be used as a novel predictor for the prediction of mortality in the old Chinese population ${ }^{17)}$. Chang LH et al. also demonstrated that UTCC was associated with CV outcomes and in patients with type 2 diabetes ${ }^{22)}$. Furthermore, our previous study revealed that UTCC was an independent predictor of long-term $\mathrm{CV}$ and all-cause mortality in patients with acute myocardial infarction ${ }^{18)}$. According to the abovementioned literature $^{17,21)}$, UT and UTCC were useful parameters for the diagnosis of PAD and could be used to evaluate the peripheral vascular function and future outcomes. Therefore, we tried to evaluate whether UTCC is also an independent predictor of long-term mortality in patients with normal ABI. Additionally, we compared the additive predictive values of UTCC, ABI, baPWV, and LVEF for CV and all-cause mortality. As we know, LVEF is regarded as an important parameter for $\mathrm{CV}$ outcome and mortality in the literature ${ }^{23-25}$. In our study, UTCC, baPWV, and LVEF are all significant and independent predictors of $\mathrm{CV}$ and all-cause mortality after multivariate analysis, but $\mathrm{ABI}$ was not. This finding might suggest that for patients with normal $A B I, A B I$

\section{Advance Publication Journal of Atherosclerosis and Thrombosis}


was not a good indicator of adverse CV events or mortality. Furthermore, we performed a nested Cox model for the prediction of $\mathrm{CV}$ and all-cause mortality. For CV mortality (Fig.2A), baPWV, UTCC, and LVEF all had additive predictive value to the basic model. Basic model + UTCC showed a better predictive value than basic model $+\mathrm{ABI}$, basic model + baPWV, and basic model $+\operatorname{LVEF}(P \leq 0.012)$. For all-cause mortality (Fig.2B), baPWV, UTCC, and LVEF all had additive predictive value to the basic model. Additionally, basic model + UTCC had a better predictive value than basic model $+\mathrm{ABI}$ and basic model $+\operatorname{LVEF}(P \leq 0.013)$. Therefore, these findings might suggest that UTCC was a better parameter than $\mathrm{ABI}$ and LVEF in predicting long-term $\mathrm{CV}$ and allcause mortality in patients with normal ABI.

Because UT and UTCC were both useful parameters for the diagnosis of $\mathrm{PAD}$ and predictors for CV outcomes ${ }^{14-18)}$, the improvement of UT or UTCC might have a good impact on future outcomes. PAD is one of the vascular bed diseases with extremely high morbidity and mortality. It also has a higher chance to have a coexistent disease in other vascular beds such as coronary artery disease and cerebrovascular disease. Arita Y et al. ever reported that after endovascular treatment for PAD, ABI, and UT had improved ${ }^{26)}$. As we know, endovascular therapy for PAD could improve the symptom of claudication, limb function, walking performance, and wound healing and decrease the risk of amputation, which might improve the survival of patients with PAD. Therefore, the improvement of UT or UTCC by endovascular therapy might improve future $\mathrm{CV}$ outcomes. Other treatment strategies that can improve UT or UTCC might also have the chance to improve CV outcomes; however, we still need further studies to investigate the issue.

\section{Study Limitations}

First, our study did not evaluate the non-fatal outcomes for patients with normal ABI. Second, we did not withdraw the CV medications because of ethical issues. However, we had adjusted the CV medications in our multivariate analysis, and the influence of medications might be reduced after the adjustment. Third, statin use was not recorded initially in our study. In our hospital, the chart was not available if patients did not visit our hospital again for more than 10 years. Therefore, the data of statin were incomplete and could not be analyzed in this study.

\section{Conclusions}

Our study is the first to investigate the usefulness of UTCC for the prediction of long-term CV and allcause mortality in patients with normal ABI. Our study revealed that UTCC is significantly associated with long-term CV and all-cause mortality in this patient group. Additionally, it has a better additive predictive value of $\mathrm{CV}$ and all-cause mortality than ABI and LVEF. Therefore, UTCC is a simple, novel, and useful parameter for identifying a high-risk patient with normal ABI.

\section{Acknowledgements}

Mortality data were provided by the Collaboration Center of Health Information Application, Ministry of Health and Welfare, Executive Yuan.

\section{Funding}

None.

\section{Competing Interests} exists.

The authors have declared no competing interest

\section{References}

1) Hasimu B, Li J, Nakayama T, Yu J, Yang J, Li X, Hu D. Ankle brachial index as a marker of atherosclerosis in chinese patients with high cardiovascular risk. Hypertens Res, 2006; 29: 23-28

2) Fowkes FG, Housley E, Cawood EH, Macintyre CC, Ruckley CV, Prescott RJ. Edinburgh artery study: Prevalence of asymptomatic and symptomatic peripheral arterial disease in the general population. Int J Epidemiol, 1991; 20: 384-392

3) Aboyans V, Ricco JB, Bartelink MEL, Björck M, Brodmann M, Cohnert T, Collet JP, Czerny M, De Carlo M, Debus S, Espinola-Klein C, Kahan T, Kownator S, Mazzolai L, Naylor AR, Roffi M, Röther J, Sprynger M, Tendera M, Tepe G, Venermo M, Vlachopoulos C, Desormais I; ESC Scientific Document Group. 2017 ESC Guidelines on the Diagnosis and Treatment of Peripheral Arterial Diseases, in collaboration with the European Society for Vascular Surgery (ESVS): Document covering atherosclerotic disease of extracranial carotid and vertebral, mesenteric, renal, upper and lower extremity arteriesEndorsed by: the European Stroke Organization (ESO)The Task Force for the Diagnosis and Treatment of Peripheral Arterial Diseases of the European Society of Cardiology (ESC) and of the European Society for Vascular Surgery (ESVS). Eur Heart J, 2018; 39: 763-816

4) Aboyans V, Desormais I, Lacroix P. Poorly compressible leg arteries: A specific presentation of peripheral artery disease. J Am Coll Cardiol, 2012; 60: 643; author reply 643-644 
5) Arain FA, Ye Z, Bailey KR, Chen Q, Liu G, Leibson CL, Kullo IJ. Survival in patients with poorly compressible leg arteries. J Am Coll Cardiol, 2012; 59: 400-407

6) Singh PP, Abbott JD, Lombardero MS, Sutton-Tyrrell K, Woodhead G, Venkitachalam L, Tsapatsaris NP, Piemonte TC, Lago RM, Rutter MK, Nesto RW, Bypass Angioplasty Revascularization Investigation 2 Diabetes Study G. The prevalence and predictors of an abnormal ankle-brachial index in the bypass angioplasty revascularization investigation 2 diabetes (bari $2 \mathrm{~d}$ ) trial. Diabetes Care, 2011; 34: 464-467

7) Adragao T, Pires A, Branco P, Castro R, Oliveira A, Nogueira C, Bordalo J, Curto JD, Prata MM. Ankle-brachial index, vascular calcifications and mortality in dialysis patients. Nephrol Dial Transplant, 2012; 27: 318325

8) Hanssen NM, Huijberts MS, Schalkwijk CG, Nijpels G, Dekker JM, Stehouwer CD. Associations between the ankle-brachial index and cardiovascular and all-cause mortality are similar in individuals without and with type 2 diabetes: Nineteen-year follow-up of a population-based cohort study. Diabetes Care, 2012; 35: 1731-1735

9) Heald CL, Fowkes FG, Murray GD, Price JF, Ankle Brachial Index C. Risk of mortality and cardiovascular disease associated with the ankle-brachial index: Systematic review. Atherosclerosis, 2006; 189: 61-69

10) Chen SC, Chang JM, Hwang SJ, Tsai JC, Liu WC, Wang CS, Lin TH, Su HM, Chen HC. Ankle brachial index as a predictor for mortality in patients with chronic kidney disease and undergoing haemodialysis. Nephrology (Carlton), 2010; 15: 294-299

11) Xu L, He R, Hua X, Zhao J, Zhao J, Zeng H, Li L, Liu F, Jia W. The value of ankle-branchial index screening for cardiovascular disease in type 2 diabetes. Diabetes Metab Res Rev, 2019; 35: e3076

12) Jimenez ZN, Pereira BJ, Romao JE, Jr., Makida SC, Abensur H, Moyses RM, Elias RM. Ankle-brachial index: A simple way to predict mortality among patients on hemodialysis--a prospective study. PLoS One, 2012; 7: e42290

13) Manzano JJ, De Silva DA, Pascual JL, Chang HM, Wong MC, Chen CP. Associations of ankle-brachial index (abi) with cerebral arterial disease and vascular events following ischemic stroke. Atherosclerosis, 2012; 223: 219-222

14) Carter SA. Indirect systolic pressures and pulse waves in arterial occlusive diseases of the lower extremities. Circulation, 1968; 37: 624-637

15) Cachovan M, Linhart J, Prerovsky I. Morphology of pulse wave curve from various segments of the lower limb in man. Angiology, 1968; 19: 381-392

16) Allen J, Overbeck K, Nath AF, Murray A, Stansby G. A prospective comparison of bilateral photoplethysmography versus the ankle-brachial pressure index for detecting and quantifying lower limb peripheral arterial disease. J Vasc Surg, 2008; 47: 794-802

17) Sheng CS, Li Y, Huang QF, Kang YY, Li FK, Wang JG. Pulse waves in the lower extremities as a diagnostic tool of peripheral arterial disease and predictor of mortality in elderly chinese. Hypertension, 2016; 67: 527-534

18) Hsu PC, Lee WH, Tsai WC, Chen YC, Chi NY, Chang CT, Chu CY, Lin TH, Lee CS, Lai WT, Sheu SH, Su HM. Upstroke time per cardiac cycle as a novel parameter for mortality prediction in patients with acute myocardial infarction. J Clin Med, 2020; 9

19) Yamashina A, Tomiyama H, Takeda K, Tsuda H, Arai T, Hirose K, Koji Y, Hori S, Yamamoto Y. Validity, reproducibility, and clinical significance of noninvasive brachial-ankle pulse wave velocity measurement. Hypertens Res, 2002; 25: 359-364

20) Tomiyama H, Yamashina A, Arai T, Hirose K, Koji Y, Chikamori T, Hori S, Yamamoto Y, Doba N, Hinohara S. Influences of age and gender on results of noninvasive brachial-ankle pulse wave velocity measurement--a survey of 12517 subjects. Atherosclerosis, 2003; 166: 303-309

21) Yu S, Lu Y, Xiong J, Teliewubai J, Chi C, Ji H, Zhou Y, Fan X, Zhang J, Blacher J, Li J, Zhang Y, Xu Y. Comparison of ankle-brachial index and upstroke time in association with target organ damage: The northern shanghai study. J Am Soc Hypertens, 2018; 12: 703-713

22) Chang LH, Hwu CM, Chu CH, Won JGS, Chen HS, Lin LY. Upstroke time per cardiac cycle is associated with cardiovascular prognosis in type 2 diabetes. Endocr Pract, 2019; 25: 1109-1116

23) Levy WC, Mozaffarian D, Linker DT, Sutradhar SC, Anker SD, Cropp AB, Anand I, Maggioni A, Burton P, Sullivan MD, Pitt B, Poole-Wilson PA, Mann DL, Packer M. The seattle heart failure model: Prediction of survival in heart failure. Circulation, 2006; 113: 1424-1433

24) Pocock SJ, Ariti CA, McMurray JJ, Maggioni A, Kober L, Squire IB, Swedberg K, Dobson J, Poppe KK, Whalley GA, Doughty RN, Meta-Analysis Global Group in Chronic Heart F. Predicting survival in heart failure: A risk score based on 39372 patients from 30 studies. Eur Heart J, 2013; 34: 1404-1413

25) Breathett K, Allen LA, Udelson J, Davis G, Bristow M. Changes in left ventricular ejection fraction predict survival and hospitalization in heart failure with reduced ejection fraction. Circ Heart Fail, 2016; 9

26) Arita Y, Ogasawara N, Hasegawa S. Correlations Between the Ankle-Brachial Index, Percentage of Mean Arterial Pressure, and Upstroke Time for Endovascular Treatment. Cardiol Res, 2020; 11: 392-397 